\title{
The Multiple-Faces configuration illusion in schizophrenic patients
}

\author{
Maria Lucia de Bustamante Simas, Christiane Delusia de Oliveira Rocha, Rafael Freitas Modesto \\ Sedycias, Viviane Ferreira do Amaral and Felipe Schuler de Menezes \\ Universidade Federal de Pernambuco, Brazil
}

\begin{abstract}
The Multiple-Faces Configuration Illusion is observed by staring at a black dot while paying attention to a face in the peripheral visual field (Simas, 2000). Changes of facial expressions, movements, depth (EMF) or different face identities (MF) are observed in this illusion. Forty participants took part in the study: 20 outpatients diagnosed with schizophrenia, and 20 people helping friends or relatives in a hospital clinic. The face-stimulus was placed in a partition stand and participants instructed to describe any visual event occurring during $90 \mathrm{~s}$, one eye at a time. Results showed that $100 \%$ of women in the experimental group (EG) reported perceiving events related to MF with the left eye. Significantly fewer women saw these events with the right eye. Also, fewer men in the experimental group and men and women in the control group saw these events with either eye. Keywords:face perception, face adaptation, schizophrenia, illusion, multiple-faces.
\end{abstract}

Received 9 June 2008; received in revised form 27 June 2008; accepted 30 June 2008. Available online 30 June 2008

\section{Introduction}

The Multiple-Faces Configuration (MFC) illusion (Simas, 2000; Simas \& Santos, 2005) is observed when a face is presented almost anywhere in peripheral vision. When this happens, changes of facial expressions, facial movements, and even tridimensional faces seem to occur within the face-stimulus outlines. If presented further, then other characteristics may appear to change, including the whole face, and faces of other identities may be perceived in continuous flashes lying over the face-stimulus. Initially thought to be observed only around the blind spot (Simas, 2000), it can be found to occur whenever a face is presented away from the fovea. It can be observed both monocularly or binocularly, and tends to persist for as long as the starring away from the face-stimulus continues.

One of our early hypotheses (Simas, 2000) was that some structures within the brain specialize in detecting faces since birth. This conjecture has been extensively investigated in the literature for many years. Sugita (2008), for instance, studied how monkeys deprived of perceiving monkey or human faces since birth react when required to discriminate between faces they never saw. Considering that their performance was very poor, the author argues for

Maria Lucia de Bustamante Simas, Christiane Delusia de Oliveira Rocha, Rafael Freitas Modesto Sedycias, Viviane Ferreira do Amaral and Felipe Schuler de Menezes, Laboratório de Percepção Visual, LabVis-UFPE, Departamento de Psicologia, Universidade Federal de Pernambuco. Correspondence regarding this article should be addressed to Maria Lucia de Bustamante Simas, Laboratório de Percepção Visual, LabVis-UFPE, Departamento de Psicologia, Universidade Federal de Pernambuco, 50.670-901, Recife, PE, Brasil. e-mail: maria.simas@pq.cnpq.br the existence of specific mechanisms for face processing that would also present a critical period during their development.

This concept of an innate mechanism to detect, discriminate, recognize and identify faces has also been investigated in human infants. Rhodes, Maloney, Turner and Ewing (2007), for instance, argue for a face tuning mechanism, operating mostly at low spatial frequencies. They did not find discrimination for the "average face" mostly used in studies involving face prototypes. Another study using evoked potentials with human infants found evidence for species face recognition (de Haan, Pascalis, \& Johnson, 2002). These studies are in agreement with our hypothesis that brain mechanisms tune for faces more frequently seen since birth, mostly for the mother face. Simas and Santos(2005) found evidence supporting that notion because the MFC illusion was more frequently seen when the face-stimulus was that of the mother of each participant as opposed to the father's or any other relative. However, Quinn, Kelly, Lee, Pascalis and Slater (2008), studying human and non-human face attractiveness with 3-4 months old infants, did not find selectivity for the human face and concluded for a general mechanism.

Our past results on the MFC illusion also point out to some contrast between the processing of facial expressions versus the perception of faces of different identities. We found support to that distinction in the literature, which suggests the involvement of different brain areas in the processing of facial expressions and face identity (Fox, Oruç, \& Barton, 2008; Posamentier, 2003; Andrews \& Ewbank, 2004; Winston, Henson, Fine-Goulden, \& Dolan, 2004). Other studies with infants also point to distinct processes of high and low spatial frequencies related to the perception of face identity and facial expressions (e.g. 
Goffaux, Hault, Michel, Vuong, \& Rossion, 2005; Goffaux \& Rossion, 2006; Winston, Vuilleumier, \& Dolan, 2003; Vuilleumier, Armony, Driver, \& Dolan, 2003). Heering, Turati, Rossion, Bulf, Goffaux and Simion (2008) argue that face perception in the new-born involves low spatial frequencies (0-0.5 cycles per visual angle), implicating holistic processing, and Heering, Houthuys and Rossion (2007) argue that holistic face perception is established in the 4 year-old child.

\section{The MFC illusion and schizophrenia}

A number of studies have shown deficits in processing of emotional expressions in schizophrenics, regardless of the illness sub-type and the presence of positive or negatives symptoms. Martin, Baudouin, Tiberghien and Franck (2005) used faces featuring different emotions by the same or different facial identities. As different emotions were displayed by the same or other identities, schizophrenic patients performed poorly in comparing emotions with the same facial identity, but even worse for same or different emotions and different facial identities. These authors found that worse performance was related to severity of negative symptoms. In a more recent study, Caharel, Bernard, Thibaut, Haouzir, Di MaggioClozel, Allio, Fouldrin, Petit, Lalonde and Rebaï (2007) working with Event Related Potentials (ERPs) found that schizophrenic patients showed deficits in both sensory (P1) and perceptual (N170) processing stages. They concluded that "these patients appear to have difficulty in encoding the structure of a face and thereby do not evaluate correctly familiarity and emotion."

\section{Previous and current experiments' methodology}

The original method used by Simas (2000; Simas \& Santos, 2005) consisted of placing solid black circles over the nose and two fixation spots on both sides of the displayed face, for viewing at about $25-30 \mathrm{~cm}$. Participants were instructed to close one of the eyes and fixate the right or left dot (depending on the tested eye) while moving the face printout forward or backward until the black circle on the nose of the printed face disappeared. Participants were asked to describe any changes they were seeing while maintaining steady fixation away from the face-stimulus. The same procedure was used for each eye. Participants were also told that events could be happening very fast. Under these conditions, participants typically describe random disappearance of the nose, of one or both eyes, mouth, hair, of either half-face, etc. Increases in the size of the eyes or the mouth are often mentioned. All those events may occur in the absence of the MFC illusion. This illusion is often preceded by some noticeable emotional expression such as a smile, an exclamation, a laugh, a reaction of surprise, etc. The time to observe the effect varies widely from one person to the other as well as from trial to trial, and depends on whether the observer has seen the effect before or not (Simas, 2000; Simas \& Santos, 2005).

Based on responses given by participants in these early experiments, we developed a system of four categories to classify reports registered either in video or audio: (a) disappearance/darkening/whitening of the eyes, nose, mouth, face, nasal face, temporal face, or hair; (b) variation in size of these face parts; (c) movement or change in facial expression of the eyes, mouth, face, or eyebrows; (d) perception of different characteristics or of other faces: 3-D, face upside down, the subject's own face, younger faces, older faces, seeing teeth, moustache, beard, hair changes, seeing profile, seeing other faces. The first category, which does not reveal the presence of the illusion, is likely to be related to the Troxler fading effect (Troxler 1804, as cited by Mennemeir, Chatterjee, Watson, Wertman, Carter, \& Heilman, 1994) where disappearances may occur in the periphery while staring takes place. We considered only reports falling in categories three and/or four as perception of the MFC illusion.

In most of our recent experiments, verbal descriptions of events taking place during the experimental sessions are being analyzed as whether or not they fit into two categories: EMF and MF. Category EMF refers to prior category three for events occurring within the face-stimulus such as changes in: (a) facial expressions; (b) facial movements or (c) perceived facial depth; whereas category MF refers to prior category four for the perception of events such as: (a) changes in the hair outline; (b) changes of gender, age or orientation, etc.; (c) new characteristics such as the outline of beard or moustache; and, more important, (d) intermittent or continuous changes of face identities, for as long as the observer wishes to stare. All of those superimposed on the face-stimulus. We also started to quantify the frequency of EMF and MF events by having participants press either one of two keyboard keys or one of two mechanical counters, each assigned to count occurrences of events related to these categories.

Despite the use of large samples in our on-going investigations on the MFC illusion (e.g. 80-192 volunteers), most participants were healthy undergraduates, or their families and friends of varying ages (18-70 yrs-old). Since the MFC illusion involves both domains, perceived changing of facial expressions and of facial identities, we are particularly interested in assessing how a mind affected by schizophrenia processes this illusion.

The present study, thus, intends to measure the MFC illusion with people diagnosed as suffering from schizophrenia. This is a multi-faceted disease that severely affects the individual's perception and cognition (refer to CID 10; DSM IV). We wanted to find out whether all participants saw the two categories, EMF and MF, or only one of them. Did the perception of EMF and MF differ between EG and CG? Also, our design allowed comparisons between groups with respect to gender or preferred eye.

\section{Screening}

Our main purpose was to investigate whether the MFC illusion was perceived in the same way by participants with or without the diagnosis of schizophrenia. Thus, we ran a screening test to select participants that saw the illusion, in order to compose an experimental (EG) and a control group (CG). For this, we followed a procedure similar 
to that of our earlier experiments (Simas, 2000; Simas \& Santos, 2005). Also, since the experiment required that all volunteers could perceive and report the MFC illusion, the exclusion criteria was not having perceived or being unable to report the illusion during this screening test.

\section{Method}

\section{The screening test}

\section{Participants}

Seventy four participants (45 in the EG and 29 in the $\mathrm{CG}$ ) performed the screening test. Fifteen females and 10 males diagnosed with schizophrenia did not meet the inclusion criteria for the EG. Among them, three saw but did not report the illusion during the experimental session, four only reported the original categories 1 and 2 , four were in no conditions to follow the instructions, four were too distracted to engage in the test, and 10 did not see or report the MFC illusion. The remaining 20 were included in the EG to participate in the present experiment. For the participants that could be included in the CG, only 9, of the 29 tested, did not report the illusion. The remaining 20 participated in the Control Group.

\section{Stimuli}

Figure 1 shows the face-stimulus consisting of a female face $\left(21.2^{\circ}-23.1^{\circ}\right.$ of visual angle) in white paper size A4, with black dots $(0.5 \mathrm{~cm}$ diameter $)$ lying over the nose and on either side of the face, depending on the eye being tested. The black dot at the nose was located at the blind spot when the distance between the participant's face and the face-stimulus was within 18.1-19.9 cm. Figure 2 shows the scheme of the stimulus presentation and Figure 3 the position of the participants during the experiment. A partition stand was used to place the stimulus.

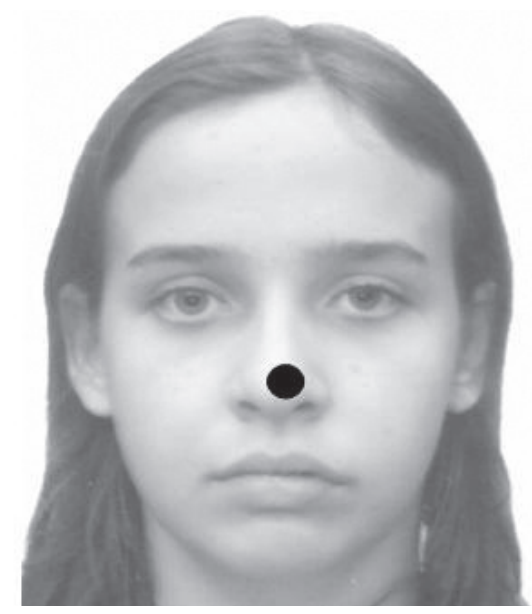

Figure 1. Face-stimulus for right or left eye.

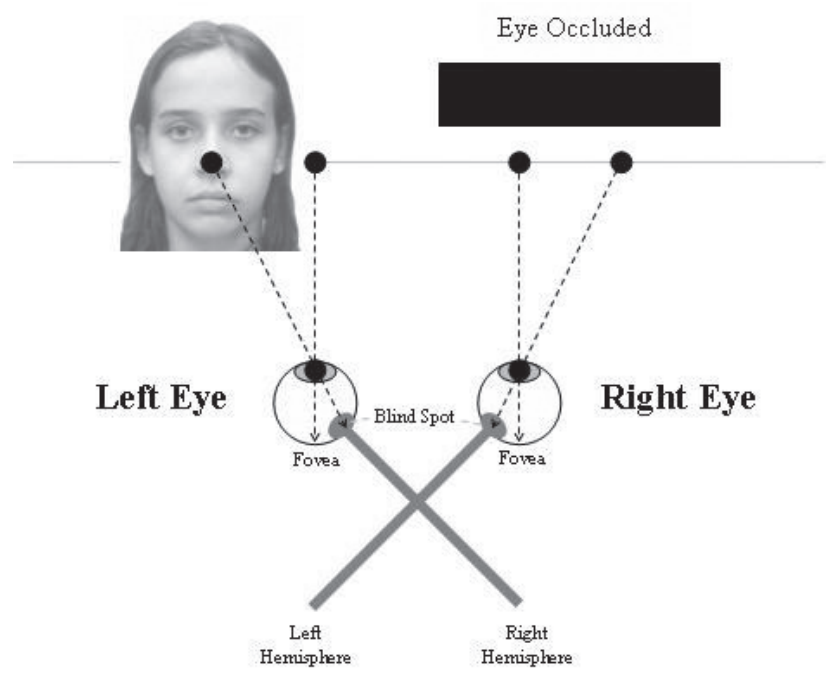

Figure 2. Schematic showing the blind spot.

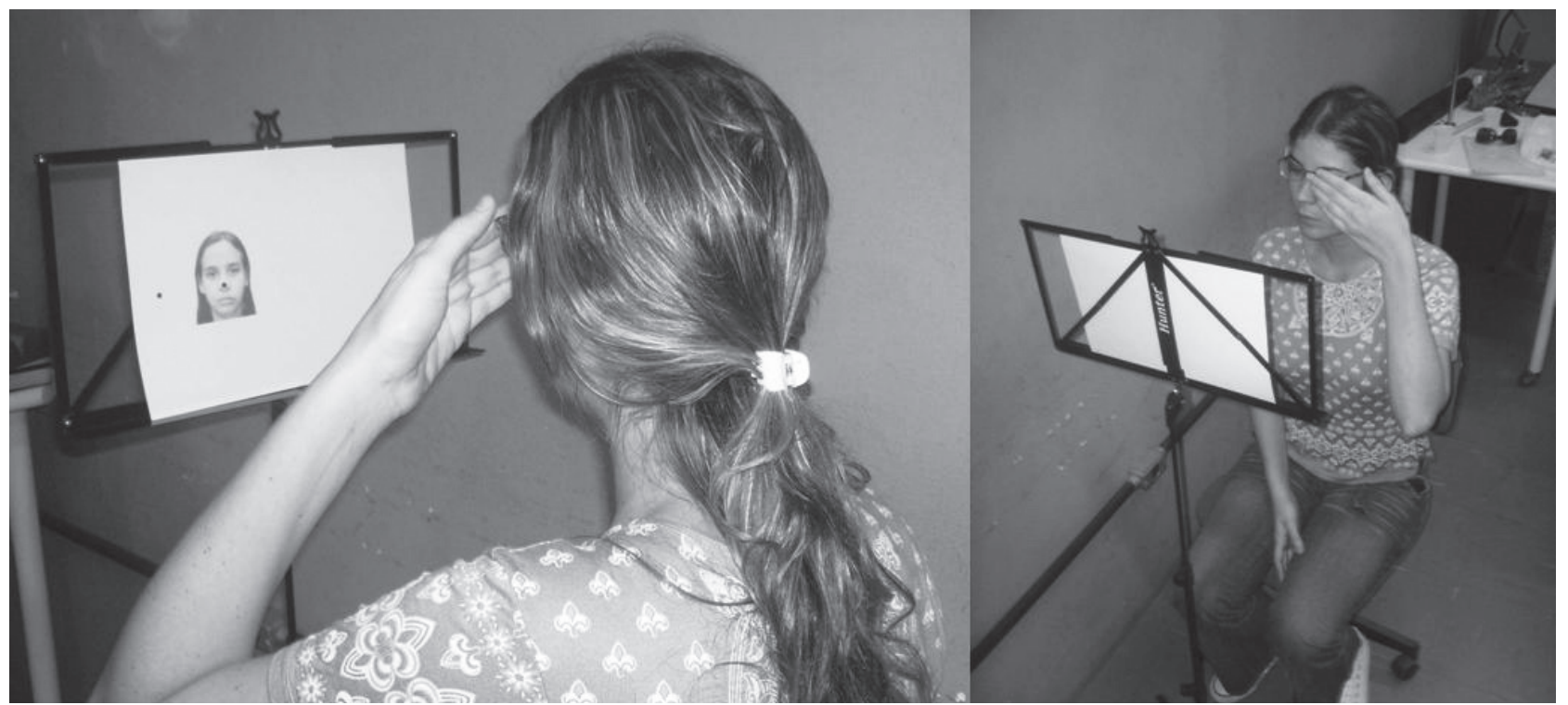

Figure 3. Position of the participants during the experiment 


\section{Procedure}

We asked the participants to sit in front of the partition stand with the stimulus while occluding (without pressing) the left eye with the left hand or vice versa, as shown in Figure 2. The participant was told to move the head forward or backward until the black dot on the nose disappeared. Then, while sustaining this distance, we instructed them to keep staring at the black dot aside of the face-stimulus during a period of $90 \mathrm{~s}$, and tell what was happening with the image, even if it were something that might be happening very fast. This was always the only instruction given. Also, we deliberately did not use words such as face, appearance, look, facade, visage, expression, features, profile, portrait, etc. Instead, we only used the word image to avoid bias in the participants' responses. When finished with one eye, the test continued with the other eye. We made written notes of the description of events given by the participants.

\section{The experiment}

In addition to the screening test, we conducted an experiment. The main purpose of the experiment was to investigate how the two categories EMF and MF were perceived by participants from the EG and CG.

\section{Participants}

There were 40 participants in this experiment, 11 males (19-62 years old) and nine females (21-52 years old) in the $\mathrm{CG}$, and 11 males (23-52 years old) and nine females (33-68 years old) in the EG. The mean age of CG and EG was 35 (SD $=9.9)$ and $40(\mathrm{SD}=9.8)$, respectively. Formal education in both groups ranged between complete elementary and incomplete high school. They had normal or corrected to normal vision.

Participants in the EG were attendees of two public outpatient clinics in Recife, Pernambuco, Brazil. These are called "Centros de Atenção Psicossocial", CAPS, and provide daily activities, medication and care to mentally ill people. Volunteers' diagnosis of schizophrenia was based on the ICD 10, and they were medicated mostly with typical antipsychotics. Only three were treated with atypical medication. Unfortunately we were not granted access to patients' anamneses and specific medication.

Participants in the CG were recruited among people that were helping patients waiting in line for medical assistance for other non-neuropsychiatric illnesses at the "Hospital das Clínicas da Universidade Federal de Pernambuco em Recife, PE, Brazil". All procedures were previously approved by the University's Ethical Committee and every subject signed a consent form.

\section{Stimuli}

We ran the experiment with the same face-stimulus and partition stand used in the screening test but, this time, also included a hand-held audio recorder.

\section{Procedure}

Originally, we intended to quantify the type (EMF or $\mathrm{MF}$ ) and frequency of the occurring events for both the EG and CG. However, the task of pressing one or two mechanical counters each time specific events occurred was found to be very difficult, mainly for participants of the EG. Consequently, we used almost the same procedure of the screening test with both the EG and CG. But this time, we asked, at the end of the experimental session, if the participant agreed to make an audio record of the answers to a few standard questions because we intended to further expose the actual contents and nature of the perceived events.

Since our design did not allow measuring the frequency of the perceived events for each participant, we only analyzed whether or not each participant saw either or both categories. Therefore, we counted as one, all of those who reported seeing any visual events related to EMF and/or MF, through either or both eyes.

Table 1. Percentage of participants who reported the Multiple-Face Configuration illusion by group, gender, category and eye. ${ }^{1}$ difference between eyes in MF was significant, $\mathrm{p}=.03 .{ }^{2}$ difference between eyes in MF was not significant, $\mathrm{p}=.05 .{ }^{3}$ difference between eyes in MF was significant, $\mathrm{p}=.01 .{ }^{4}$ difference between eyes in MF was significant, $\mathrm{p}<.001 .{ }^{5}$ difference between eyes in MF was significant, $\mathrm{p}<.001$

\begin{tabular}{|c|c|c|c|c|c|c|c|c|c|}
\hline \multirow{2}{*}{ Category } & & \multicolumn{2}{|c|}{ EMF } & \multicolumn{2}{|c|}{$\mathrm{MF}$} & \multicolumn{2}{|c|}{$E M F+M F$} & \multicolumn{2}{|c|}{ COLOR } \\
\hline & & $\mathrm{RE}$ & LE & $\mathrm{RE}$ & LE & $\mathrm{RE}$ & LE & $\mathrm{RE}$ & LE \\
\hline & Female $\mathrm{N}=9$ & $33 \%$ & $33 \%$ & $67 \%{ }^{1}$ & $100 \% \%^{1,2,3,45}$ & $67 \%$ & $100 \%$ & $56 \%$ & $56 \%$ \\
\hline \multicolumn{10}{|l|}{$\mathrm{EG}$} \\
\hline & Male $N=11$ & $36 \%$ & $64 \%$ & $73 \%^{2}$ & $64 \%$ & $100 \%$ & $82 \%$ & $36 \%$ & $27 \%$ \\
\hline$p$-value & & & & & 0,0298 & 0,0268 & & & \\
\hline & Female $\mathrm{N}=9$ & $56 \%$ & $44 \%$ & $56 \%{ }^{3}$ & $44 \%{ }^{4}$ & $78 \%$ & $56 \%$ & $22 \%$ & $11 \%$ \\
\hline \multicolumn{10}{|l|}{$\mathrm{CG}$} \\
\hline & Male $N=11$ & $45 \%$ & $73 \%$ & $45 \%{ }^{5}$ & $45 \%{ }^{5}$ & $73 \%$ & $82 \%$ & $27 \%$ & $36 \%$ \\
\hline $\mathrm{p}$-value & & & & & & & & & \\
\hline
\end{tabular}


Table 2. Percentage of participants who reported the Multiple-Face Configuration Illusion by gender, category and eye.

\begin{tabular}{|c|c|c|c|c|c|c|c|c|c|}
\hline \multirow{2}{*}{\multicolumn{2}{|c|}{ Category }} & \multicolumn{2}{|c|}{ EMF } & \multicolumn{2}{|c|}{$\mathrm{MF}$} & \multicolumn{2}{|c|}{$\mathrm{EMF}+\mathrm{MF}$} & \multicolumn{2}{|c|}{ COLOR } \\
\hline & & $\mathrm{RE}$ & LE & $\mathrm{RE}$ & LE & $\mathrm{RE}$ & LE & $\mathrm{RE}$ & LE \\
\hline $\mathrm{EG}$ & Female $\mathrm{N}=9$ & $33 \%$ & $33 \%$ & $67 \%$ & $100 \%$ & $67 \%$ & $100 \%$ & $56 \%$ & $56 \%$ \\
\hline CG & Female $\mathrm{N}=9$ & $56 \%$ & $44 \%$ & $56 \%$ & $44 \%$ & $78 \%$ & $56 \%$ & $22 \%$ & $11 \%$ \\
\hline $\mathrm{p}-\mathrm{va}$ & & & & & 0.0088 & & 0.0193 & & 0.0301 \\
\hline $\mathrm{EG}$ & Male $N=11$ & $36 \%$ & $64 \%$ & $73 \%$ & $64 \%$ & $100 \%$ & $82 \%$ & $36 \%$ & $27 \%$ \\
\hline \multicolumn{10}{|l|}{$C G$} \\
\hline & Male $N=11$ & $45 \%$ & $73 \%$ & $45 \%$ & $45 \%$ & $73 \%$ & $82 \%$ & $27 \%$ & $36 \%$ \\
\hline $\mathrm{p}-\mathrm{va}$ & & & & & & .04 & & & \\
\hline
\end{tabular}

\section{Results}

Tables 1, 2 and 3 show percentages of participants (by group, gender and eye) that saw illusory visual events consistent with categories EMF and MF. Tables 4-6 show direct comparisons between eyes for categories EMF and MF in percentages. All differences between and within samples were tested statistically with the proportion test.

Thus, we found that more women from the EG reported events related to $\mathrm{MF}$ with the LE than the men of the same group (EG), $(p=.03)$, as well as the women, $(p=.01)$, andman, $(p=.01)$, belonging to the $\mathrm{CG}$, regardless of the eye (refer to Table 1). The difference between the LE and the RE for females in the EG was also significant $(p=.04)$. When both categories were taken together, $\mathrm{EMF}+\mathrm{MF}, 100 \%$ women in the EG reported events related to the illusion with the LE, contrary to $100 \%$ men of the same group that did so with the $\operatorname{RE}(p=.03)$. Considering both groups across gender, participants in the $\mathrm{EG}$ reported more $\mathrm{MF}$ related events through the $\operatorname{LE}(p=.01)$, (refer to Table 3). Comparing reports of perceiving colors by groups and gender, more females in the EG reported seeing color than females in the $\mathrm{CG}(p=.03)$.
We also made direct comparisons between eyes by group, gender and category. Table 4 shows prevalence of the LE for reports of MF related events by females belonging to the EG. This was true when compared to all reports of EMF by females in EG $(p=.01)$, reports of MF by males of the same group ( $p$ $=.03)$, and reports by females of CG $(p=.01)$. On the other hand, more males in the EG reported MF rather than EMF when viewing through the RE ( $p=.05$ ), (refer to Tables 4 and 5). Finally, significantly more participants in the EG, across gender, reported perceiving events related to MF with the LE than those in the $\mathrm{CG}(p=.01)$, also, there were more reports of MF than EMF with the RE in the EG $(p=.02)$. No significant differences were observed between categories or eyes in the Control Group (refer to Table 6).

\section{Discussion}

Every woman of the group diagnosed with schizophrenia reported seeing events compatible with MF and with the LE. Unexpectedly, this differed significantly, regardless of the eye, from the $64 \%$ of men in the EG, as well as from $44 \%$ and $45 \%$ of women and men, respectively, in the CG that reported so. This finding was not expected because our research with participants not suffering from this disease has

Table 3. Percentage of participants who reported the Multiple-Face Configuration Illusion by group.

\begin{tabular}{|c|c|c|c|c|c|c|c|c|}
\hline \multirow{2}{*}{ Category } & \multicolumn{2}{|c|}{ EMF } & \multicolumn{2}{|c|}{$\mathrm{MF}$} & \multicolumn{2}{|c|}{$\mathrm{EMF}+\mathrm{MF}$} & \multicolumn{2}{|c|}{ COLOR } \\
\hline & $\mathrm{RE}$ & $L E$ & $\mathrm{RE}$ & LE & $\mathrm{RE}$ & LE & $\mathrm{RE}$ & LE \\
\hline $\mathrm{EG} \mathrm{N}=20$ & $35 \%$ & $50 \%$ & $70 \%$ & $80 \%$ & $85 \%$ & $90 \%$ & $45 \%$ & $40 \%$ \\
\hline $\mathrm{CGN}=20$ & $50 \%$ & $60 \%$ & $50 \%$ & $45 \%$ & $75 \%$ & $70 \%$ & $25 \%$ & $25 \%$ \\
\hline$p$-value & & & & 0.01 & & & & \\
\hline
\end{tabular}


Table 4. Percentage of participants who reported the Multiple-Face Configuration Illusion by group, gender, category and eye. ${ }^{1}$ Significant interactions, $\mathrm{p}=.05 .{ }^{2}$ Significant interactions, $\mathrm{p}=.03$.

\begin{tabular}{|c|c|c|c|c|c|}
\hline \multirow{2}{*}{ Category } & & \multicolumn{2}{|c|}{ EMI } & \multicolumn{2}{|c|}{ MF } \\
\hline & & $\mathrm{RE}$ & LE & $\mathrm{RE}$ & LE \\
\hline & Female $\mathrm{N}=9$ & 33 & 67 & $33^{2}$ & $100^{2}$ \\
\hline \multicolumn{6}{|l|}{$\mathrm{EG}$} \\
\hline & Male $\mathrm{N}=11$ & $36^{1}$ & $73^{1}$ & $64^{2}$ & $64^{2}$ \\
\hline & Female $N=9$ & 56 & 56 & 44 & 44 \\
\hline \multicolumn{6}{|l|}{$\mathrm{CG}$} \\
\hline & Male $N=11$ & 45 & 45 & 73 & 45 \\
\hline
\end{tabular}

shown a tendency towards predominance of the RE in at least two experiments involving size and eccentricity (manuscript in preparation). We announce it here, but it is still too early to be able to state that as a fact. Most of the other effects reinforce this findings (refer to Tables 4, 5 and 6).

While studying the MFC illusion, we hypothesized that at least two different mechanisms were activated by it (Simas, 2000). One mechanism initially seems to act locally (i.e. for eyes, mouth, nose, moustache, etc.), and would be related to our category EMF, and later, another mechanism appears to act globally (i.e. whole faces, hair, beard, etc.), and would be related to our category MF. These hypotheses are consistent with the literature that compares performance in processing facial expressions and facial identities (e.g. Fox et al., 2008; Caharel et al., 2007; Martin et al., 2005), more specifically, in the case of schizophrenic patients' facial emotions. Numerous studies in the literature have shown that schizophrenic patients perform poorly in the processing of facial emotion (e.g. Martin et al., 2005). We understand that this relates to facial expressions and, therefore, to our category EMF in the MFC illusion. So, it is interesting that the present

Table 5. Percentage of participants who reported the Multiple-Face Configuration Illusion by gender, group, category and eye. ${ }^{1}$ Significant interactions, $\mathrm{p}=.01 .{ }^{2}$ Significant interactions, $\mathrm{p}=.01$.

\begin{tabular}{|c|c|c|c|c|c|}
\hline \multirow{2}{*}{\multicolumn{2}{|c|}{ Category }} & \multicolumn{2}{|c|}{ EMF } & \multicolumn{2}{|c|}{ MF } \\
\hline & & \multirow{2}{*}{$\frac{\mathrm{RE}}{33}$} & \multirow{2}{*}{$\frac{L E}{67}$} & \multirow{2}{*}{$\frac{\mathrm{RE}}{33^{1}}$} & \multirow{2}{*}{$\frac{\mathrm{LE}}{100^{1,2}}$} \\
\hline $\mathrm{EG}$ & Female $\mathrm{N}=9$ & & & & \\
\hline $\mathrm{CG}$ & Female $\mathrm{N}=9$ & 56 & 56 & $44^{2}$ & $44^{2}$ \\
\hline$E G$ & Male $N=11$ & 36 & 73 & 64 & 64 \\
\hline $\mathrm{CG}$ & Male $N=11$ & 45 & 45 & 73 & 45 \\
\hline
\end{tabular}


Table 6. Percentage of participants who reported the Multiple-Face Configuration Illusion by group, category and eye. ${ }^{1}$ Significant interactions, $\mathrm{p}=.02 .{ }^{2}$ Significant interactions, $\mathrm{p}=.01$.

\begin{tabular}{lcccccc}
\hline \multirow{2}{*}{ Category } & \multicolumn{2}{c}{ EMF } & & \multicolumn{2}{c}{ MF } \\
\cline { 2 - 3 } \cline { 5 - 6 } EG N=20 & RE & LE & & RE & LE \\
\hline CG N=20 & $35^{1}$ & $70^{1}$ & & 50 & $80^{2}$ \\
\hline
\end{tabular}

study suggests a tendency to show a smaller number of male and female participants in the EG reporting EMF in comparison with the CG (Table 6). Although these differences were not significant, they might show up in larger samples.

Schizophrenic patients also have difficulty in processing identities. Martin and colleagues (2005), for instance, have shown that people with this diagnosis have difficulty differentiating identities and distinct emotions. Different identities with the same emotion, the same identity with different emotions and different identities with different emotions are some of the tasks in which schizophrenic patients perform very poorly. Therefore, it is interesting and unexpected that more schizophrenic patients report seeing other faces than the control group, despite the small sample size. We found that this was true only for women in the EG (not men in the EG) and through the LE. The task, however, does not demand face recognition, but the participants do perceive and report different identities. The preference for the LE would mean a higher involvement of the right hemisphere.

These findings have strong implications for the research in laterality, differences attributed to gender, stimulus configuration changes, among others. Therefore, we consider these as the beginning of an investigation that deserves considerable attention. Finally, it is noteworthy that for the first time the participants claimed to see color during the experiment, but not necessarily faces in color; this opens a new direction in our research.

\section{Acknowledgments}

This research was supported by the Conselho Nacional de Desenvolvimento Científico e Tecnológico, CNPq, Grant \#30.0856/2005-9

\section{References}

Andrews, T.J., Ewbank, M.P. (2004). Distinct representations for facial identity and changeable aspects of faces in the human temporal lobe. Neuroimage 23, 905-913.
American Psyquiatric Association. (1995). Manual de diagnóstico e estatística dos transtornos mentais - DSM IV.

Caharel, S., Bernard, C., Thibaut, F., Haouzir, S., Di Maggio-Clozel, C., Allio G., Fouldrin, G., Petit, M., Lalonde, R., \& Rebaï, M. (2007). The effects of familiarity and emotional expression on face processing examined by ERPs in patients with schizophrenia. Schizophrenia Research, 95, 186-196.

de Haan, M., Pascalis, O., \& Johnson, M. H. (2002). Specialization of neural mechanisms underlying face recognition in human infants. Journal of Cognitive Neuroscience.14, 2, 199-209.

de Heering A, Turati C, Rossion B, Bulf H, Goffaux V, \& Simion F. (2008). Newborns' face recognition is based on spatial frequencies below 0.5 cycles per degree. Cognition, 106, 444-454.

de Heering A, Houthuys S, \& Rossion B. (2007). Holistic face processing is mature at 4 years of age: evidence from the composite face effect. Journal of Experimental Child Psychology, 96, 1, 57-70.

Desimone, R. (1991). Face-selective cells in the temporal cortex of monkeys. Journal of Cognitive Neuroscience, 3, 1-8.

Fox, C.J., Oruç, I., \& Barton, J.J.S. (2008). It doesn't matter how you feel. The facial identity aftereffect is invariant to changes in facial expression. Journal of Vision, 8, 3, 1-13.

Goffaux, V., Hault, B., Michel, C., Vuong, Q. C., \& Rossion, B. (2005). The respective role of low and high spatial frequencies in supporting configural and featural processing of faces. Perception, 34, 77-86.

Goffaux, V., \& Rossion, B. (2006). Faces are "spatial"--holistic face perception is supported by low spatial frequencies. Journal of Experimental Psychology: Human Perception and Performance, 32, 1023-1039.

Martin, F., Baudouin, J. Y., Tiberghien, G., \& Franck, N. (2005). Processing emotional expression and facial identity in schizophrenia. Psychiatry Research, 134, 143-153.

Mennemeir, M. S., Chatterjee, A., Watson, R. T., Wertman, E., Carter, L. P., \& Heilman, K. M. (1994). Contributions of the parietal and frontal lobes to sustained attention and habituation. Neuropsychologia, 32, 703-716.

Organização Mundial de Saúde. (1993). Classificação internacional de doenças problemas relacionados à saúde: CID 10. São Paulo: EDUSP.

Posamentier, M.T., \& Abdi, H. (2003). Processing faces and facial expressions Neuropsychology Review, 13, 113-143.

Quinn, P.C., Kelly, D.J., Lee, K., Pascalis, O., \& Slater, A.M. (2008). Preference for attractive faces in human infants extends beyond conspecifics. Developmental Science, 11, 1, 76-83.

Rhodes, G., Maloney, L.T., Turner, J., \& Ewing, L. (2007). Adaptive face coding and discrimination around the average face. Vision Research, 47, 974-989.

Simas, M. L. B. (2000). The multiple-faces phenomenon: some investigative studies. Perception, 29, 1393-1396.

Simas, M. L. B. Santos, N. A. (2005). O fenômeno de muitas-faces. Psicologia Reflexão e Crítica, 18, 104-108.

Sugita, Y. (2008). Face perception in monkeys reared with no exposure to faces. Proceedings of the National Academy of Sciences of the United States of America, 105, 1, 394-398.

Troxler, D. (1804). Uë ber das Verschwinden gegebener Gegensta«nde innerhalb unseres Gesichtskreises. Ophthalmologie, volume 2, Eds K Himly, J A Smidt (Jena: Fromann) PP. 51-53.

Vuilleumier. P., Armony, J. L., Driver, J., \& Dolan, R. J. (2003). Distinct spatial frequency sensitivities for processing faces and emotional expressions. Natural Neuroscience, 6, 624-631.

Winston, J. S., Henson, R. N., Fine-Goulden, M. R., \& Dolan, R. J. (2004). fMRI-adaptation reveals dissociable neural representations of identity and expression in face perception. Journal of Neurophysiology, 92, 3, 1830-1839.

Winston, J. S., Vuilleumier, P., \& Dolan, R. J. (2003). Effects of lowspatial frequency components of fearful faces on fusiform cortex activity. Current Biology, 13, 1824-1829. 